\title{
A New Fuzzy-Based Maximum Power Point Tracker for a Solar Panel Based on Datasheet Values
}

\author{
Ali Kargarnejad, ${ }^{1}$ Mohsen Taherbaneh, ${ }^{2}$ and Amir Hosein Kashefi ${ }^{3}$ \\ ${ }^{1}$ Department of Computer, South Tehran Branch, Islamic Azad University, No. 209, North Iranshahr St., \\ P.O. Box 11365/4435, Tehran, Iran \\ ${ }^{2}$ Department of Electrical and Information Technology, Iranian Research Organization for Science and Technology (IROST), \\ Azadegan Highway, Ahmad Abad Mostavfi, Enghelab St., Ehsanirad St., Tehran 3353136846, Iran \\ ${ }^{3}$ Young Researchers Club, South Tehran Branch, Islamic Azad University, No. 173, Near of Karim Khan Bridge, \\ Sepahbod Gharani Avenue, Tehran 1584743311, Iran \\ Correspondence should be addressed to Mohsen Taherbaneh; m.taherbaneh@irost.org
}

Received 11 November 2012; Revised 23 January 2013; Accepted 30 January 2013

Academic Editor: Ching-Song Jwo

Copyright (C) 2013 Ali Kargarnejad et al. This is an open access article distributed under the Creative Commons Attribution License, which permits unrestricted use, distribution, and reproduction in any medium, provided the original work is properly cited.

Tracking maximum power point of a solar panel is of interest in most of photovoltaic applications. Solar panel modeling is also very interesting exclusively based on manufacturers data. Knowing that the manufacturers generally give the electrical specifications of their products at one operating condition, there are so many cases in which the specifications in other conditions are of interest. In this research, a comprehensive one-diode model for a solar panel with maximum obtainable accuracy is fully developed only based on datasheet values. The model parameters dependencies on environmental conditions are taken into consideration as much as possible. Comparison between real data and simulations results shows that the proposed model has maximum obtainable accuracy. Then a new fuzzy-based controller to track the maximum power point of the solar panel is also proposed which has better response from speed, accuracy and stability point of view respect to the previous common developed one.

\section{Introduction}

Nowadays, using low-cost energy sources in all industries is of interest. Renewable energy sources are suitable options to cover this request, where photovoltaic energy is one of them. The main problems of this source of energy are low energy conversion efficiency and high installation cost. Many researches have been done regarding increasing the efficiency of a solar cell. Consequently, different kinds of solar cells have been designed and introduced. Knowing that a solar panel is one of the essential parts of a photovoltaic system which converts solar energy to electrical energy, it has also nonlinear $I-V$ characteristic curves. Modeling of a photovoltaic system predicts the system's electrical behavior in various environmental and load conditions. In order to reach accurate prediction of the system's electrical behavior, it is needed to have comprehensive and precise models for all parts of the system especially their solar panels. Meanwhile, because of nonregulating voltage of a solar panel, a DCDC converter is generally utilized to regulate this voltage in photovoltaic systems. As mentioned, because of high installation cost of a photovoltaic system, it is an obligation that the system shall contain high efficiency parts. Hence, a maximum power point tracker (MPPT) is generally used in photovoltaic systems. Different kinds of MPPTs have been introduced and developed. Fuzzy logic is one of the suitable methods to find the maximum power point (MPP) of a solar panel which has good stability and high response rate.

In this research, a new fuzzy-based MPPT for a photovoltaic system is introduced and implemented. In addition, a novel and accurate model for a solar panel is also developed only based on manufacturer's data. There are two general developed models for a solar cell/panel: one-diode and twodiode models. The one-diode model requires lower amount of available data from the manufacturers which is suitable for this study. In the literature, many papers have been published 
regarding the solar cell/panel modeling and maximum power point tracking.

Reference [1] introduces two separate models for a solar cell, where dependency of the models parameters on environmental conditions has not been fully developed. Hence, the models are not accurate sufficiently. References [2,3] develop a solar panel model based on datasheet values with several considerations. Series and shunt resistances of the model have been assumed constant, and their dependencies on environmental conditions have been ignored. Furthermore, dark-saturation current of the model has been described as a dependent variable on temperature, and its dependency on irradiance has been also neglected. Reference [4] presents an analytical one-diode model for a solar panel. Series and shunt resistances of the model have been stated by the slopes at the open-circuit and short-circuit points of the solar panel's $I$ $V$ curves, respectively. Dependency of the model parameters on environmental conditions has been briefly expressed. Therefore, the model is not suitable for applications which need high accuracy.

Reference [5] uses one-diode model to evaluate three popular types of solar panels: thin film and multi-and monocrystalline silicon. In the model, shunt resistance has been considered infinite. The dark-saturation current has been also considered to be dependent only on the temperature. A circuit-based simulation model for a solar cell has been demonstrated in [6]. The interaction between a proposed power converter and the photovoltaic array has been also studied. In order to extract the initial values of the model parameters at standard conditions, it has been assumed that the slope of current-voltage curve in opencircuit point is available from the manufacturers. Clearly, this parameter is not generally supported by a solar panel datasheet, and it can be obtained only through experiment.

It is worth noting that the first goal of this study is introducing a model for a solar panel with maximum obtainable accuracy using only datasheet values. Meanwhile, the darksaturation current of the proposed model is considered to be dependent on either irradiance or temperature. Dependencies of the other model parameters on environmental conditions are also developed accurately as much as possible. The second goal of this research is developing a new fuzzybased MPPT. There are also many published researches regarding fuzzy-based MPPT. Reference [7] proposes a fuzzy-based MPPT for a photovoltaic system under variable temperature and insolation conditions. Simulation results show that the system with fuzzy-based MPPT increases the efficiency of energy production from the system without MPPT. Reference [8] tests the performance of fuzzy logic with various membership functions to optimize the MPPT. Results show that the performance of fuzzy logic MPPT with five membership functions is better than fuzzy-based MPPT with three membership functions. Reference [9] presents a wide range of fuzzy-based MPPTs for PV sizing and efficiency improvement. Hence, the rest of the paper is organized as follows.

Section 2 of this paper is devoted to the derivation of nonlinear mathematical expressions for one-diode model. Newton's method is chosen to solve the nonlinear model

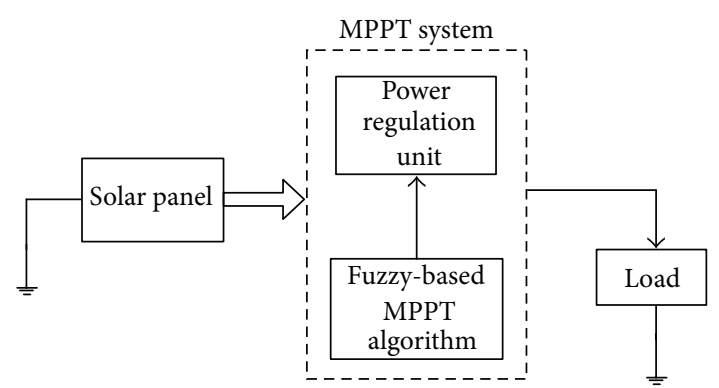

FIGURE 1: Block diagram of the proposed constant power regulator.

equations. A modification in the solving approach is introduced to have the best convergence. Extraction of the initial values of the model parameters is given based on data provided by manufacturers. Then, the accurate description of the model's parameters is developed from their dependencies on environmental conditions point of view. Boost converter and fuzzy-based MPPT are also designed and developed in this section. Section 3 provides simulation results and their interpretation. Several figures are presented which show the behavior of the model parameters and the fuzzy-based tracking algorithm. A measurement system is also developed in order to provide a test bed for investigation of the proposed model in this section. A comparison among experimental data, simulation results of the proposed model, and data from the model by the other authentic references is also presented in this section. Detailed conclusion on the results of the research is provided in Section 4.

\section{Study Method}

The aim of this research is delivering maximum output power of a solar panel in any environmental conditions. In addition, regulating the output power of the solar panel is considered instead of regulation its output voltage. Hence, a PV converter with capability of providing maximum achievable output power is designed and implemented. This regulated PV converter can be also used in power subsystem of a satellite between power generation unit (solar array) and power conversion unit (DC-DC converter). Figure 1 shows the block diagram of the proposed system for this study, where all parts of the system are reviewed and modeled.

2.1. Solar Panel Model. In this research, one-diode model for a solar cell is used based on datasheet values which provided equations quantify the mentioned model briefly. Figure 2 shows equivalent circuit of the one-diode model for a solar cell.

The general current-voltage characteristic of the model is given as

$$
i=I_{\mathrm{ph}}-I_{0}\left(e^{\left(v+i R_{s}\right) / V_{T}}-1\right)-\frac{v+i R_{s}}{R_{p}}, \quad V_{T}=\frac{n k T}{q} .
$$

The model has five unknown parameters: $I_{\mathrm{ph}}, I_{0}, n, R_{s}$, and $R_{p}$. As we know, a solar panel is composed of parallel 


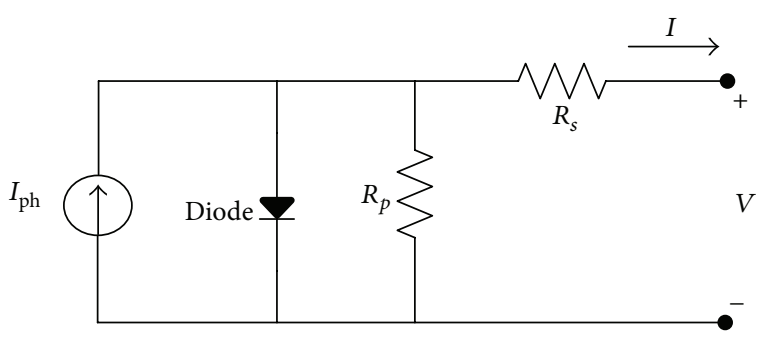

FIGURE 2: Equivalent circuit of one-diode model for a solar cell.

combination of several cell strings, and a string contains several cells in series. So, a one-diode model can be developed for a solar panel. The one-diode model for a solar panel will be developed based on datasheet values exclusively without using any measurements. Manufacturers of solar panels typically provide their product's electrical parameters at only one operating standard condition, while in some applications, their electrical behaviors in other environmental conditions are of interest. Meanwhile, solar panels operate over a wide range of environmental conditions; however, the manufacturers' information is not sufficient to determine their overall performance. Consequently, to fulfill this requirement, a suitable model for a solar panel is of interest. It is assumed that a solar panel is composed of $n_{p}$ strings, each string with $n_{s}$ series cells. Equation (1) could be expanded in order to describe the general current-voltage characteristic for a solar panel [1]. Consider

$$
i=I_{\mathrm{ph}}-n_{p} I_{0}\left(e^{\left(v+i R_{s}\right) / a}-1\right)-\frac{v+i R_{s}}{R_{p}}, \quad a=\frac{n_{s} n k T}{q} .
$$

As discussed earlier, the five unknown parameters $I_{\mathrm{ph}}$, $I_{0}, a, R_{s}$, and $R_{p}$ are also needed to describe the model for a solar panel. In order to extract the model parameters, (2) is examined in three operating points; short circuit, open circuit, and maximum power point (MPP). Equations (3)(5) explain the model's behavior in the mentioned points. As the derivative of power in MPP is zero, so, the fourth relation can be expressed by derivative of (2). Then, the derivative is examined at MPP and it leads to (6). Consider

$$
\begin{gathered}
I_{\mathrm{sc}}=I_{\mathrm{ph}}-n_{p} I_{0} e^{I_{\mathrm{sc}} R_{s} / a}-\frac{I_{\mathrm{sc}} R_{s}}{R_{p}} \quad \text { (short circuit), } \\
I_{\mathrm{oc}}=0=I_{\mathrm{ph}}-n_{p} I_{0} e^{V_{\mathrm{oc}} / a}-\frac{V_{\mathrm{oc}}}{R_{p}} \quad \text { (Open circuit), } \\
I_{\mathrm{mpp}}=I_{\mathrm{ph}}-n_{p} I_{0} e^{\left(V_{\mathrm{mpp}}+I_{\mathrm{mpp}} R_{s}\right) / a}-\frac{V_{\mathrm{mpp}}+I_{\mathrm{mpp}} R_{s}}{R_{p}} \quad(\mathrm{MPP}) \\
I_{\mathrm{mpp}}+V_{\mathrm{mpp}} \frac{-\left(\left(n_{p} I_{0} / a\right) e^{\left(V_{\mathrm{mpp}}+I_{\mathrm{mpp}} R_{s}\right) / a}+1 / R_{p}\right)}{1+R_{s}\left(\left(n_{p} I_{0} / a\right) e^{\left(V_{\mathrm{mpp}}+I_{\mathrm{mpp}} R_{s}\right) / a}+1 / R_{p}\right)}=0 .
\end{gathered}
$$

In order to define the last required relation, there are two ways. In $[2,4,5]$, slope of an $I-V$ curve in short circuit point has been considered as model's parallel resistance. Some others have used the temperature coefficients reported by manufacturers [3]. In this research, the slope of $I-V$ curves in short circuit point is also employed which results in

$$
\frac{-1}{R_{p}}=\frac{-\left(\left(n_{p} I_{0} / a\right) e^{I_{\mathrm{sc}} R_{s} / a}+1 / R_{p}\right)}{1+R_{s}\left(\left(n_{p} I_{0} / a\right) e^{I_{\mathrm{sc}} R_{s} / a}+1 / R_{p}\right)} .
$$

In this research, series and shunt resistances of the model are also taken into account invariant because of limitation in manufacturers' data. Using data provided by the manufacturers in standard conditions, the five nonlinear equations are solved, and the model becomes definite in the standard conditions (irradiance $=1000 \mathrm{~W} / \mathrm{m}^{2}$; temperature $=25^{\circ} \mathrm{C}$ ). Newton's method is chosen to solve the following nonlinear equations [10]:

$$
\begin{aligned}
& R=f_{i}\left(I_{\mathrm{ph}}, I_{0}, a, R_{s}, R_{p}\right)=0, \quad i=1,2, \ldots, 5, \\
& J=\operatorname{Jacobian}(R)=\frac{\partial\left(f_{1}, \ldots, f_{5}\right)}{\partial\left(I_{\mathrm{ph}}, I_{0}, a, R_{s}, R_{p}\right)} .
\end{aligned}
$$

In this research, to have convergence in solving the five aforementioned nonlinear equations, a modification in the solving approach is proposed. To solve the equations, a starting point $x_{0}=\left[I_{\mathrm{ph}}, I_{0}, a, R_{s}, R_{p}\right]$ must be determined, and both matrixes $R$ and $J$ are also examined at that point. Then, $\delta x$ is described based on (9), and, consequently, (10) states the new estimation of the root of the equations. Consider

$$
\begin{gathered}
J^{k} \delta x^{k}=-R^{k}, \\
x_{\text {new }}=x_{\text {old }}+\delta x .
\end{gathered}
$$

The previous iteration is repeated by the new start point $\left(x_{\text {new }}\right)$ until error is less than an acceptable level. Finally, it was seen that to attain an appropriate convergence, a modification coefficient $(0<\alpha<1)$ is needed to add to (10). Consider

$$
x_{\text {new }}=x_{\text {old }}+\alpha \times \delta x \text {. }
$$

The modified approach has good response to solve the model equations for the solar panel by tuning the proposed coefficient.

2.1.1. The Extraction of Initials Model Parameters. The model equations and the solving approach are implemented in MATLAB environment, and initial values of the unknown parameters are extracted in standard condition. Table 1 reports electrical specifications of the under investigation solar panel at standard conditions based on datasheets.

Table 2 provides the extracted initial values of the model parameters in standard condition based on the implemented solving nonlinear equations approach. In order to predict the solar panels behavior in different environmental conditions other than this one, the model parameters must become known. So, the model parameters dependencies on environmental conditions should be stated as this will be reported in the next section. 
TABLE 1: Datasheet information of the solar panel $\left(G=1000 \mathrm{~W} / \mathrm{m}^{2}\right.$; $\left.T=25^{\circ} \mathrm{C}\right)$.

\begin{tabular}{lc}
\hline Specification & Value \\
\hline$I_{\text {sc }}(\mathrm{A})$ & 2.98 \\
$V_{\mathrm{oc}}(\mathrm{V})$ & 20.5 \\
$I_{\mathrm{mpp}}(\mathrm{A})$ & 2.73 \\
$V_{\mathrm{mpp}}(\mathrm{V})$ & 16.5 \\
$P_{\mathrm{mpp}}(\mathrm{W})$ & 45 \\
$n_{s}$ & 36 \\
$n_{p}$ & 1 \\
$k_{i}\left(\% /{ }^{\circ} \mathrm{C}\right)$ & 0.07 \\
$k_{v}\left(\% /{ }^{\circ} \mathrm{C}\right)$ & -0.038 \\
\hline
\end{tabular}

TABLE 2: Extracted the model parameters of the solar panel in standard conditions.

\begin{tabular}{lc}
\hline Model parameters & Value \\
\hline$I_{\mathrm{ph}}(\mathrm{A})$ & 2.9811 \\
$I_{0}(\mathrm{~A})$ & $4.7401 \times 10^{-7}$ \\
$a$ & 1.3105 \\
$R_{s}(\Omega)$ & 0.2262 \\
$R_{p}(\Omega)$ & 611.5891 \\
\hline
\end{tabular}

\subsubsection{The Model Parameters Dependency on Environmental} Conditions. Solar panel manufacturers generally give electrical and thermal specifications of their products only in standard conditions. These standard data are used in the modeling of a solar panel to define the model's parameters. Nevertheless, expressing dependencies of the model's parameters on environmental conditions is not possible. In this research, dependencies of the open-circuit voltage and dark-saturation current on environmental conditions can be extracted with the most realizable accuracy based on datasheet values as follows.

Short-circuit and photo-generated currents have linear relationships with irradiance which are stated as follows [1$3]$ :

$$
\begin{gathered}
I_{\mathrm{sc}}(G)=I_{\mathrm{sc}}\left(G_{0}\right) \times \frac{G}{G_{0}}, \\
I_{\mathrm{ph}}(G)=I_{\mathrm{ph}}\left(G_{0}\right) \times \frac{G}{G_{0}} .
\end{gathered}
$$

The short-circuit current has also an independent relation with temperature as follows [1-3]:

$$
I_{\mathrm{sc}}(T)=I_{\mathrm{sc}}\left(T_{0}\right)\left(1+\frac{k_{i}}{100}\left(T-T_{0}\right)\right)
$$

Therefore, (14) states comprehensive relation of the shortcircuit current on environmental conditions, where $k_{i}$ is temperature coefficient of $I_{\mathrm{sc}}$ and it is reported by the manufacturers. Consider

$$
I_{\mathrm{sc}}(G, T)=I_{\mathrm{sc}}\left(G_{0}, T_{0}\right) \times \frac{G}{G_{0}} \times\left(1+\frac{k_{i}}{100}\left(T-T_{0}\right)\right) .
$$

The previous equation can be also used to describe the dependency of photo-generated current on environmental conditions [1-3]. To obtain dark-saturation current and open-circuit voltage dependencies on environmental conditions, (3) and (4) can be used. Reference [8] reports the dark-saturation current dependent on both irradiance and temperature as stated in (15). This equation is taken from (4) with infinite the model's parallel resistance. Consider

$$
I_{0}(G, T)=\frac{I_{\mathrm{ph}}(G, T)}{e^{V_{\mathrm{oc}}(T) / V_{T}}-1} .
$$

Dark-saturation current is also expressed only dependent on temperature in $[6,9]$ as follows:

$$
I_{0}(T)=I_{0}\left(T_{1}\right)\left(\frac{T}{T_{1}}\right)^{3 / A} e^{-\left(q E_{g} / n k\right)\left(1 / T_{1}-1 / T\right)} .
$$

In [2], combination of (3) and (4) has been used to describe the dependency of dark-saturation current on environmental conditions. It has assumed that the darksaturation current is only dependent on temperature as described in (17), while some factors in the equation are not dependent only on the temperature, and some corrections are needed. Consider

$$
I_{0}(T)=\left(I_{\mathrm{sc}}(T)-\frac{V_{\mathrm{oc}}(T)-I_{\mathrm{sc}}(T) R_{s}}{R_{p}}\right) e^{-V_{\mathrm{oc}}(T) / n_{s} V_{T}} .
$$

In this study, using (3) and (4), the dependency of the dark-saturation current on irradiance and temperature is fully developed. Consider

$$
I_{0}(G, T)=\frac{\left(I_{\mathrm{sc}}(G, T)-\left(V_{\mathrm{oc}}(G, T)-I_{\mathrm{sc}}(G, T) R_{s}\right) / R_{p}\right)}{n_{p}\left(e^{V_{\mathrm{oc}}(G, T) / a}-e^{I_{\mathrm{sc}}(G, T) R_{s} / a}\right)} .
$$

Using (4) leads to open-circuit voltage dependency on irradiance in standard temperature $\left(T_{0}\right)$. Consider

$$
\begin{aligned}
& V_{\mathrm{oc}}\left(G, T_{0}\right) \\
& \quad=a \ln \left(\frac{I_{\mathrm{ph}}\left(G, T_{0}\right) R_{p}-V_{\mathrm{oc}}\left(G, T_{0}\right)}{n_{p} I_{0}\left(G, T_{0}\right) R_{p}}\right), \quad a=\frac{n_{s} n k T_{0}}{q} .
\end{aligned}
$$

Two recursive nonlinear equations (18) and (19) are also solved with the modified Newton's approach to define the dark-saturation current and open-circuit voltage in any irradiance level but at standard temperature. Then, (20) describes the open-circuit voltage dependency on any environmental conditions $(G, T)$. Consider

$$
\begin{aligned}
V_{\mathrm{oc}}(G, T)= & V_{\mathrm{oc}}\left(G, T_{0}\right)+k_{v}\left(T-T_{0}\right) \Longrightarrow \\
V_{\mathrm{oc}}(G, T)= & \ln \left(\frac{I_{\mathrm{ph}}\left(G, T_{0}\right) R_{p}-V_{\mathrm{oc}}\left(G, T_{0}\right)}{n_{p} I_{0}\left(G, T_{0}\right) R_{p}}\right) \\
& \times a+k_{v}\left(T-T_{0}\right), \quad a=\frac{n_{s} n k T_{0}}{q},
\end{aligned}
$$




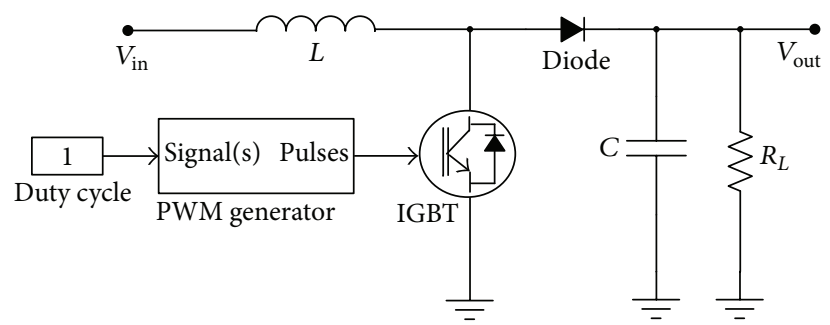

FIGURE 3: Circuit diagram of the boost converter.

where $k_{v}$ is temperature coefficient of $V_{\text {oc }}$ that is also reported by the manufacturers. Consequently, with putting the obtained $V_{\mathrm{oc}}(G, T)$ in (18), the dark-saturation current is also stated in any arbitrary environmental conditions. Therefore, using all the mentioned relevant equations, the model is completed based on datasheet values exclusively. Consider

$$
\begin{aligned}
i= & I_{\mathrm{ph}}(G, T)-n_{p} I_{0}(G, T)\left(e^{\left(v+i R_{s}\right) / a}-1\right), \\
& -\frac{v+i R_{s}}{R_{p}}, \quad a=\frac{n_{s} n k T}{q} .
\end{aligned}
$$

2.2. Boost Converter. Figure 3 shows a utilized boost converter to deliver maximum accessible power from the solar panel to the load.

Using (22), main components of the circuit are chosen [11] as follows:

$$
\begin{gathered}
D_{\text {min }}=1-\frac{V_{\text {in max }}}{V_{\text {out } \min }}, \quad D_{\text {max }}=1-\frac{V_{\text {in min }}}{V_{\text {out max }}}, \\
L>\frac{D * V_{\text {in }} *(1-D)}{f * 2 * I_{\text {out }}}, \\
C>\frac{I_{\text {out }}}{V_{\text {ripple }} * f},
\end{gathered}
$$

where $D, f$, and $V_{\text {ripple }}$ are duty cycle, switching frequency, and output voltage ripple, respectively. In this study, based on the solar panel's specifications (Table 1), the following initial data for the circuit are given:

$$
8 \mathrm{~V}<V_{\text {in }}<22 \mathrm{~V}, 30<V_{\text {out }}<150 \mathrm{~V}, V_{\text {ripple-max }}=0.3 \mathrm{~V} \text {. }
$$

The optimum values of the $L(10 \mathrm{mH})$ and $C(5 \mu \mathrm{F})$ are chosen for the converter to have the enough speed in tracking MPP. Figure 4 shows an example of the converter's electrical behavior when the solar panel is in standard condition $(G=$ $1000 \mathrm{~W} / \mathrm{m}^{2}$ and $T=25^{\circ} \mathrm{C}$ ), and $V_{\text {in }}=20.5 \mathrm{~V}$ (open-circuit condition). It can be seen that the converter needs at least 5 minute to reach the steady state condition.

Generally, in most of developed fuzzy MPPT, two parameters $d P / d I$ and its variations $\Delta(d P / d I)$ are used as fuzzy inputs. In developed model in this research, $\Delta(d P / d I)$ is not a suitable parameter, and another parameter is selected. The input variables of the proposed fuzzy controller are

$$
\Delta V=V_{i}-V_{\max }, \quad \Delta P=P_{i}-P_{\max },
$$

TABLE 3: Maximum power point tracking fuzzy rule base.

\begin{tabular}{lllccccc}
\hline$\Delta P$ & & & \multicolumn{5}{c}{$\Delta V$} \\
& NB & NM & NS & Z & PS & PM & PB \\
\hline B & NB & NM & NS & Z & PS & PS & PM \\
M & NB & NM & NS & Z & PS & PM & PM \\
S & NB & NM & Z & Z & Z & PM & PB \\
\hline
\end{tabular}

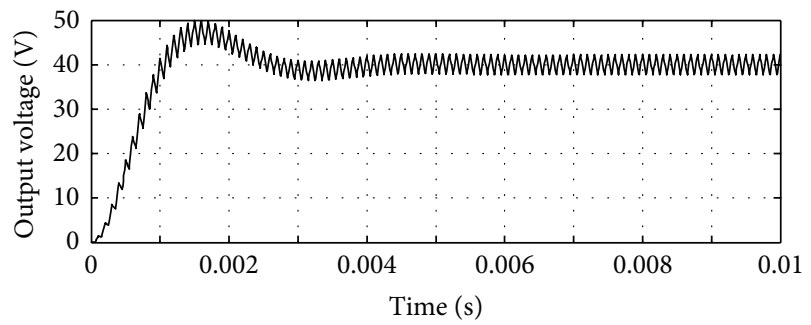

FIGURE 4: Electrical behavior of the boost converter.

where $V_{i}$ and $P_{i}$ are solar panel's voltage and power, respectively. The fuzzy controller works as follows. If $V_{i}$ is far from the $V_{\mathrm{MPP}}$, then the fuzzy controller employs only one input $(\Delta V)$, and when the $V_{i}$ is close to the $V_{\text {MPP }}$, then the other parameter $\Delta P$ is also employed and the fuzzy controller will be with the two inputs. This controller has better response from accuracy and stability points of view with respect to the previous common developed one. Table 3 shows fuzzy rule base which is used in this research.

2.3. Fuzzy-Based MPPT. $\triangle V$ and the output are expressed by seven linguistic variables: PB (positive big), PM (positive medium), PS (positive small), Z (zero), NS (negative small), NM (negative medium), and NB (negative big). $\Delta P$ is expressed only by three linguistic variables: B (big), Z (zero), and $S$ (small). In this study, triangle membership function subsets are chosen, and membership functions boundaries are expressed based on the solar panel's specifications and empirical experiments.

\section{Simulation and Experimental Results}

The developed equations in Section 2 are implemented in MATLAB environment to obtain the dependency of the solar panel's model parameters in different environmental conditions. In the first step, the dependencies of dark-saturation current and open-circuit voltage are extracted based on the manufacturer's data. Figure 5 shows the extracted darksaturation current of the solar panel in different environmental conditions but in logarithmic form. Results show that the dark-saturation current depends on both the temperature and irradiance but with more dependency on the temperature. Meanwhile, it can be seen that the dark-saturation current increases by an increase in the irradiance or the temperature in a nonlinear way.

Figure 5 also shows that, at $T=0^{\circ} \mathrm{C}$ and $G=1000 \mathrm{~W} / \mathrm{m}^{2}$, dark-saturation current has $11.1 \%$ increasing rate with respect to its value at the same temperature but in $G=100 \mathrm{~W} / \mathrm{m}^{2}$. 


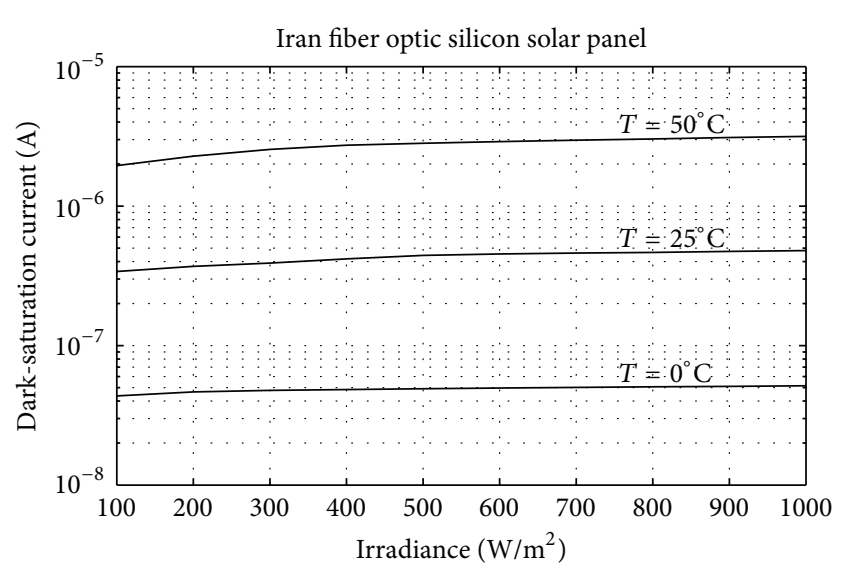

Figure 5: Dark-current saturation of the solar panel under investigation.

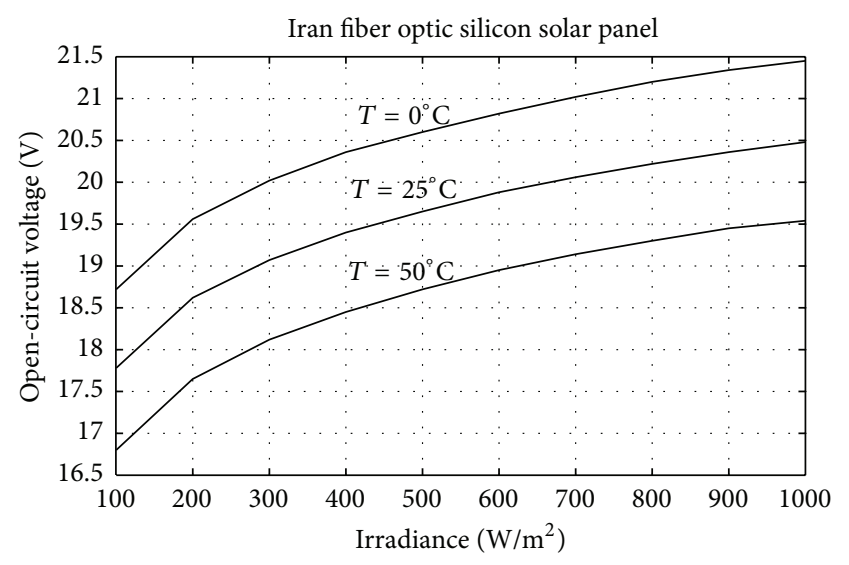

FIGURE 6: Open-circuit voltage of the solar panel under investigation.

The increasing rates for $25^{\circ} \mathrm{C}$ and $50^{\circ} \mathrm{C}$ and at the same level of changing irradiance $\left(+900 \mathrm{~W} / \mathrm{m}^{2}\right)$ are $26.1 \%$ and $36.7 \%$, respectively. These conclusions indicate that the darksaturation current depends on both the temperature and irradiance. Figure 6 presents the extracted open-circuit voltage of the solar panel in different environmental conditions using recursive equations (18), (19), and (20). Results show that the open-circuit voltage also depends on both the temperature and irradiance. Meanwhile, it can be seen that the opencircuit voltage decreases by an increase in the temperature or a decrease in the irradiance in a nonlinear way.

AT $T=0^{\circ} \mathrm{C}$ and $G=1000 \mathrm{~W} / \mathrm{m}^{2}$, open-circuit voltage has $3.82 \%$ increasing rate with respect to its value at the same temperature but in $G=500 \mathrm{~W} / \mathrm{m}^{2}$. The increasing rates for $25^{\circ} \mathrm{C}$ and $50^{\circ} \mathrm{C}$ and at the same level of changing irradiance $\left(+500 \mathrm{~W} / \mathrm{m}^{2}\right)$ are $4 \%$ and $4.17 \%$, respectively.

3.1. Measurement System. Evaluation of the proposed model should be performed by different $I-V$ solar panel curves in different environmental conditions. For this purpose, a suitable measurement system is developed and implemented.
TABLE 4: Environmental conditions of the $I-V$ solar panel curves.

\begin{tabular}{lccc}
\hline Curves & $\begin{array}{c}\text { Irradiance } \\
\left(\mathrm{W} / \mathrm{m}^{2}\right)\end{array}$ & $\begin{array}{c}\text { Temperature } \\
\left({ }^{\circ} \mathrm{C}\right)\end{array}$ & $\begin{array}{c}\text { Maxim power } \\
(\mathrm{W})\end{array}$ \\
\hline 1 & 746.4 & 38.7 & 28.12 \\
2 & 780.8 & 37 & 29.9 \\
3 & 599.5 & 44.1 & 21.72 \\
4 & 900.8 & 34.9 & 35.2 \\
5 & 946.3 & 40.9 & 35.7 \\
6 & 775.5 & 20.5 & 34.8 \\
7 & 241.6 & 31.7 & 9.6 \\
8 & 454.9 & 37.8 & 17.6 \\
9 & 850.1 & 25.4 & 36.2 \\
10 & 478 & 14.2 & 22.12 \\
\hline
\end{tabular}

A block diagram of the designed and implemented measurement system is shown in Figure 7. For the purpose of this study, extracting the $I-V$ curves will be done in a wide range of environmental conditions. Different levels of received solar irradiance were achieved by changing the solar panel orientation in horizontal and vertical directions through control of two DC motors. In order to cover a wide range of temperature, the measurements were carried out in different days of the year. The solar irradiance and temperature were also measured during the extraction of an $I-V$ curve to make sure that the environmental conditions have remained constant.

In order to extract $I-V$ curves, it is sufficient to change the panel current from zero (open circuit) to its maximum value (short circuit) continuously or step by step. Then, the characteristic curves can be obtained by measuring the corresponding voltages and currents. Hence, a variable load is required across the solar panel's output ports. In this research, the drain-source resistance of a MOSFET is used as a variable load which is controlled by its gate-source voltage [12]. The schematic diagram for the implemented electronic load is shown in Figure 8.

3.2. Validation of the Proposed Model. In this research, a multicrystalline solar panel is used to evaluate the proposed model. It is needed to evaluate the model's accuracy in a wide range of environmental conditions. For this purpose, ten extracted $I-V$ curves by the measurement system are used for this investigation which cover a wide range of irradiance and temperature as shown in Table 4. Irradiance and temperature are measured by pyranometer and temperature sensor in the measurement system. Estimated maximum power points are considered as a benchmark for the evaluation. A comparison is also done between experimental data and simulation results of the proposed model. The model introduced in [2] is also implemented to have more accurate comparison. Table 5 shows extracted real MPP and estimated MPP by our proposed model and the other model which has been introduced in [2].

Results show that the maximum and minimum errors for our proposed model are $6.2 \%$ and $1.2 \%$, respectively, 


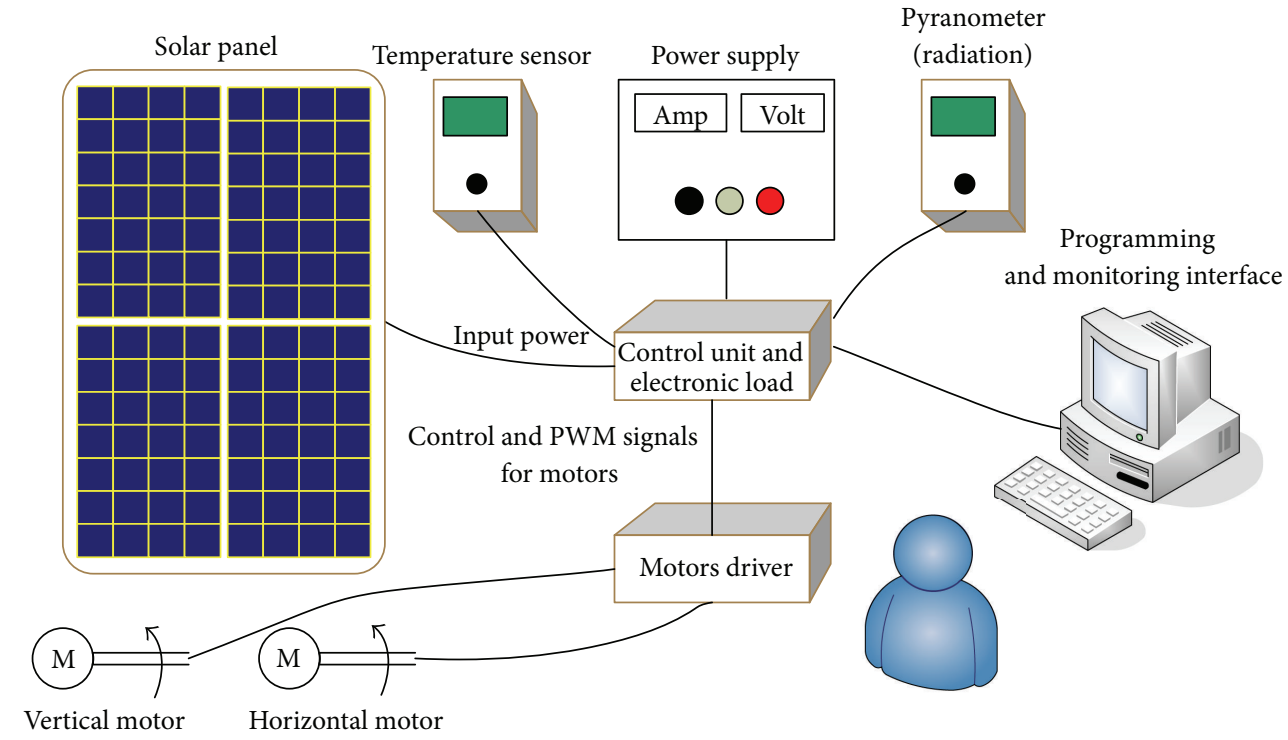

FIGURE 7: The proposed measurement system block diagram.

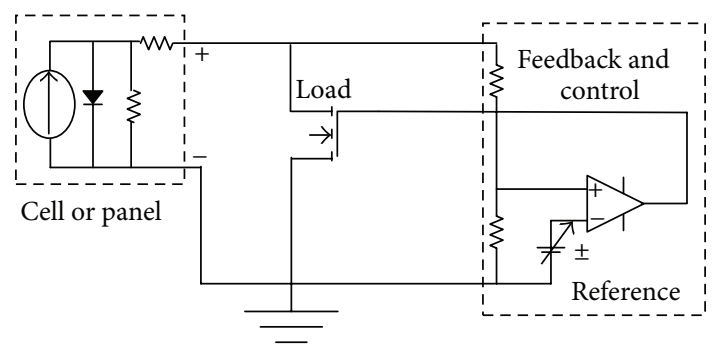

FIGURE 8: The proposed electronic load.

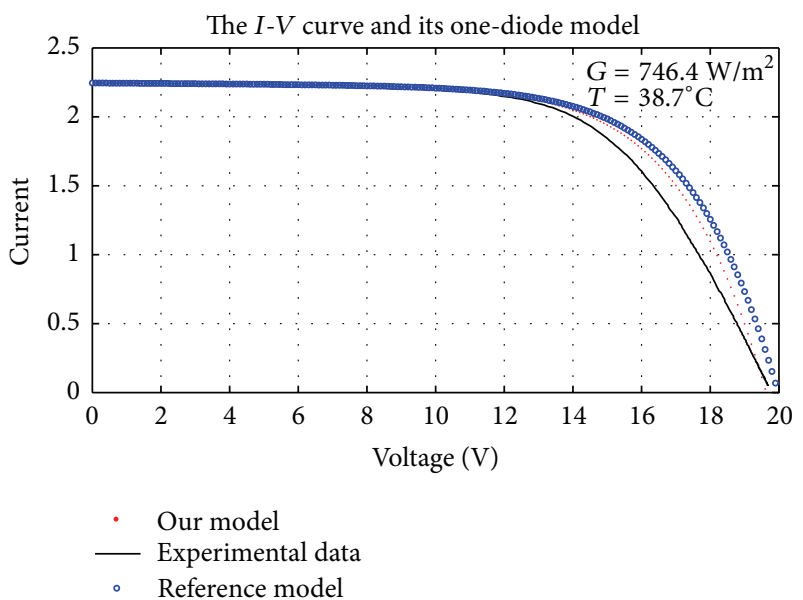

Figure 9: The solar panel $I$ - $V$ curve and its one diode models.

in estimation of MPP, while the mentioned errors for the introduced model in [2] are $9.4 \%$ and $3.2 \%$, respectively. Results show that the average error on peak power estimation in our developed model is $2.5 \%$, while it is $6 \%$ for the introduced model by [2]. As mentioned, the main goal of
TABLE 5: Maximum power point of the solar panel.

\begin{tabular}{lccccc}
\hline Curves & \multicolumn{5}{c}{ Maximum power (W) } \\
& $\begin{array}{c}\text { Experimental } \\
\text { results }\end{array}$ & $\begin{array}{c}\text { Our } \\
\text { model }\end{array}$ & Error (\%) & $\begin{array}{c}\text { Ref. } \\
\text { model }\end{array}$ & Error (\%) \\
\hline 1 & 28.12 & 29.13 & 3.6 & 29.8 & 6.0 \\
2 & 29.9 & 31.02 & 6.2 & 31.62 & 8.3 \\
3 & 21.72 & 21.97 & 1.2 & 22.9 & 5.4 \\
4 & 35.2 & 36.77 & 4.7 & 37.06 & 5.3 \\
5 & 35.7 & 36.68 & 2.7 & 36.83 & 3.2 \\
6 & 34.8 & 36.1 & 3.7 & 36.74 & 5.6 \\
7 & 9.6 & 9.21 & -4.1 & 10.23 & 6.6 \\
8 & 17.6 & 17.31 & -1.6 & 18.39 & 4.5 \\
9 & 36.2 & 37.85 & 4.6 & 38.3 & 5.8 \\
10 & 22.12 & 23.08 & 4.3 & 24.21 & 9.4 \\
\hline
\end{tabular}

this research is development of a comprehensive one-diode model for a solar panel exclusively based on datasheet values. Hence, the developed model shall be compared with the other developed one-diode models, and comparison with developed two-diode models is not the aim of this research. The developed model is valid for Si-based solar panels, where one-diode model can show their electrical behavior. It can be seen that our proposed model simulates the solar panel better than the model in which dark-saturation current is only dependent on temperature $[2,3]$. The reason of much error of the two models in comparison with experimental data is the limitation in datasheet values.

Figure 9 shows one of the extracted $I-V$ curves and the corresponding models in a specified environmental condition. Obviously, conformity of the models with real data can be seen. This procedure was done for the all extracted $I-V$ curves, and in all of them, our proposed model had more compatibility with real data. 


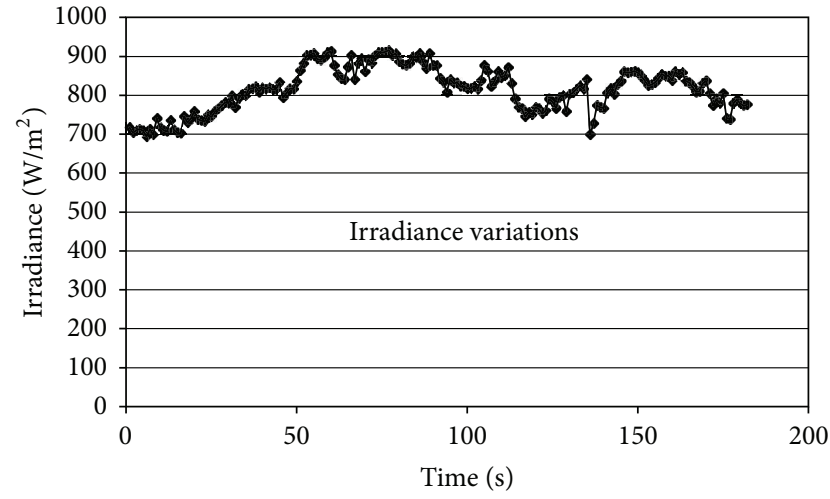

(a)

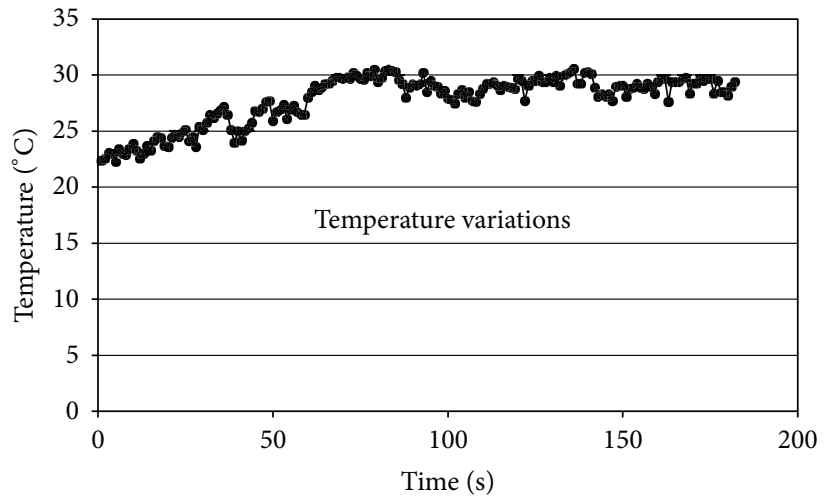

(b)

FIGURE 10: Solar irradiance and temperature variations.

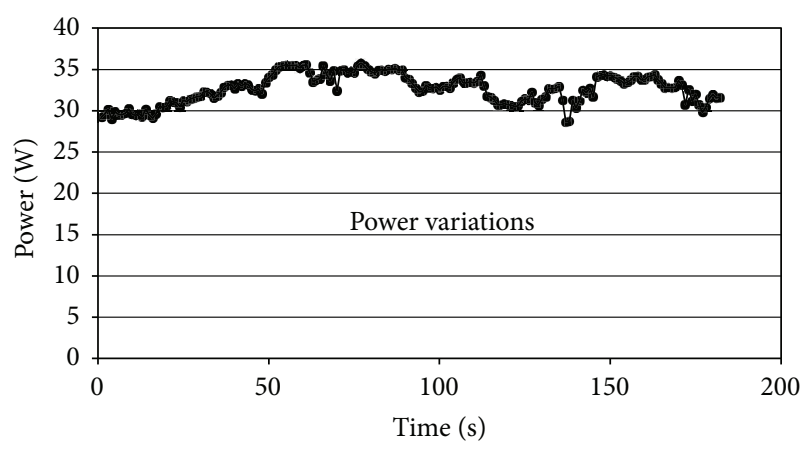

(a)

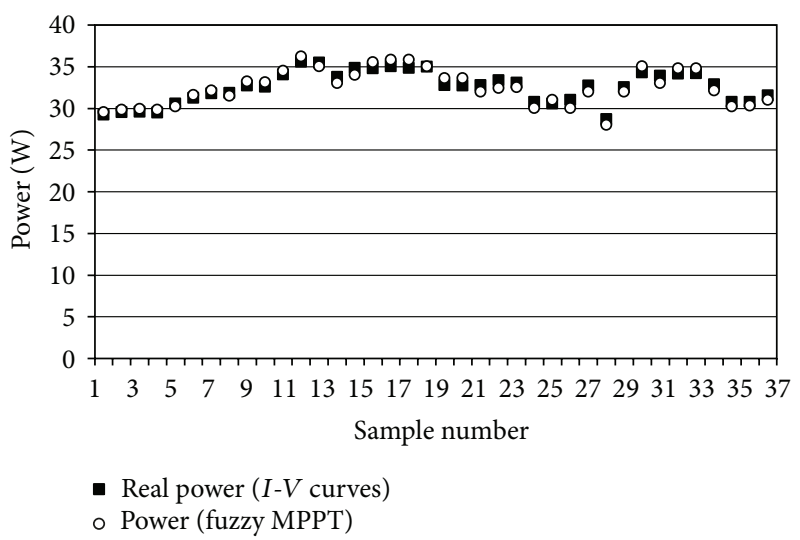

(b)

FIGURE 11: Tracking the MPP using the fuzzy controller.

3.3. Fuzzy System Results. There are many interesting observations that can be reached from this research. First, speed tracking of the developed fuzzy-based algorithm was compared with two common MPPT methods; incremental conductance and the perturbation and observation (two points) which both of them also were implemented. The test was done to find the MPPT at $T=36^{\circ} \mathrm{C}$ and $G=830 \mathrm{~W} / \mathrm{m}^{2}$. It was observed that the proposed fuzzy-based MPPT algorithm reached its maximum level after 0.57 seconds, which is a reasonable rate. The other two algorithms (incremental conductance and the perturbation and observation) reached the MPP during $1.92 \mathrm{~s}$ and $4.36 \mathrm{~s}$, respectively. It concludes that the fuzzy-based MPPT is faster than the two common methods.

Second, several $I$ - $V$ curves extracted were used to evaluate the fuzzy proposed algorithm. Figure 10 shows variations of the solar irradiance (between 702 and $915 \mathrm{~W} / \mathrm{m}^{2}$ ) and the temperature (between 22 and $30^{\circ} \mathrm{C}$ ) of these curves. Figure 11(a) shows extracted MPP of each $I-V$ curve in relevant irradiance and temperature.
The proposed fuzzy algorithm was tested for thirty-eight of the $I-V$ curves where results are shown in Figure 11(b). Block points show the solar panel maximum power points extracted from the real $I-V$ curves, whereas the white points show estimated MPPs by the proposed fuzzy algorithm. It is observed that the mentioned algorithm leads to the maximum power delivery to the load in any environmental conditions with acceptable error. Since the developed solar panel's model in this research has some error (maximum 6\%) in defining solar panel's electrical behavior, the developed fuzzy-based algorithm estimates the MPP with the mentioned error. If more accurate solar panel's model is used, the proposed fuzzy-based algorithm will have better accuracy in estimating MPP.

Third, the proposed fuzzy algorithm was tested in an abrupt change of solar irradiance (from 800 to $200 \mathrm{~W} / \mathrm{m}^{2}$ and back within 5 seconds at temperature $25^{\circ} \mathrm{C}$ ). Using the developed model for the solar panel, maximum power points in the two environmental conditions were extracted. Figure 12 shows speed tracking response of the proposed 


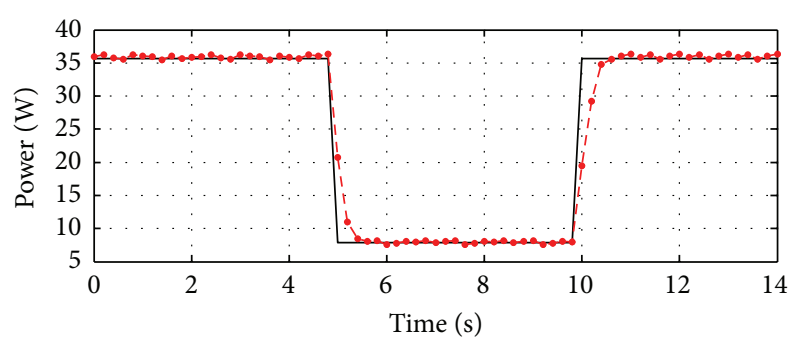

- Power from the developed model

- - Power by proposed fuzzy algorithm

FIGURE 12: Tracking speed of the proposed fuzzy-based MPPT in abrupt variation of irradiance.

fuzzy-based MPPT. It can be seen that the algorithm takes about one second to reach MPP in abrupt condition of irradiance which is a suitable speed in tracking MPP.

In high power system, as there is more than one solar panel (series or in parallel), the proposed fuzzy-based algorithm can be used if a multipoint maximum power point tracker is used to ensure that all solar panels are in MPP. For nonohmic load such as a battery which has fixed voltage, an MPPT charger in current mode shall be used instead of the utilized boost converter.

\section{Conclusion}

In this paper, a comprehensive one-diode model for a solar panel using exclusive data supported by the manufacturers was fully developed and implemented with enough accuracy. A new coefficient $(0<\alpha<1)$ was introduced for modification of Newton's method to solve the model's nonlinear equations and in order to have the best convergence. Through deep analysis, $\alpha=0.01$ was obtained as an appropriate coefficient for solving the solar panel equations. Determination of initial values of the model's parameters was done based on solving the nonlinear equations at standard conditions. Then, darksaturation current and open-circuit voltage dependencies on environmental conditions were taken into consideration with maximum accuracy as much as possible. Two recursive nonlinear equations were introduced to model the dependencies of dark-saturation current and open-circuit voltage on environmental conditions. Simulation results showed that both the dark-saturation current and open-circuit voltage depend on environmental conditions. Validation of the proposed model was done based on several extracted $I-V$ curves in a wide range of environmental conditions. According to the estimated MPPs by the proposed model and a reference model developed in [2] and in comparison with experimental data, it is concluded that the maximum error of the proposed model was $6.2 \%$ but for the reference model was $9.2 \%$. So, the introduced model can simulate a solar panel with more accuracy.

Then, a new fuzzy-based algorithm was proposed to track maximum power point of the solar panel, and it was compared with the incremental conductance and perturbation and observation methods from speed tracking point of view.
The results showed that the proposed fuzzy controller tracks the MPP within $0.57 \mathrm{~s}$, while it was $1.92 \mathrm{~s}$ and $4.36 \mathrm{~s}$ for both mentioned algorithms, respectively. The proposed fuzzy algorithm was tested in 38 points with different irradiances and temperatures. It is observed that the mentioned algorithm leads to the maximum power delivery to the load in any environmental conditions.

\section{Acknowledgment}

The authors are thankful to Islamic Azad University, South Tehran Branch, for the financial support of the following research project: "Design and implementation of neuro-fuzzy controller for maximum power point tracking of a solar panel."

\section{References}

[1] L. Castaner and S. Silvestre, Modeling Photovoltaic Systems Using Pspice, John Wiley \& Sons, 2002.

[2] D. Sera, R. Teodorescu, and P. Rodriguez, "PV panel model based on datasheet values," in Proceedings of the IEEE International Symposium on Industrial Electronics (ISIE '07), pp. 23922396, June 2007.

[3] W. De Soto, S. A. Klein, and W. A. Beckman, "Improvement and validation of a model for photovoltaic array performance," Solar Energy, vol. 80, no. 1, pp. 78-88, 2006.

[4] A. N. Celik and N. Acikgoz, "Modelling and experimental verification of the operating current of mono-crystalline photovoltaic modules using four- and five-parameter models," Applied Energy, vol. 84, no. 1, pp. 1-15, 2007.

[5] R. Chenni, M. Makhlouf, T. Kerbache, and A. Bouzid, "A detailed modeling method for photovoltaic cells," Energy, vol. 32, pp. 1724-1730, 2007.

[6] J. A. Gow and C. D. Manning, "Development of a photovoltaic array model for use in power-electronics simulation studies," IEE Proceedings, vol. 146, no. 2, pp. 193-200, 1999.

[7] M. M. Algazar, H. AL-monier, H. A. EL-halim, and M. E. E. $\mathrm{K}$ Salem, "Maximum power point tracking using fuzzy logic control," Electrical Power and Energy Systems, vol. 39, pp. 21-28, 2012.

[8] C. S. Chin, P. Neelakantan, H. P. Yoong, and K. T. K. Teo, "Optimisation of fuzzy based maximum power point tracking in PV system for rapidly changing solar irradiance," Global Journal of Technology and Optimisation, vol. 2, no. 2, pp. 130113, 2011.

[9] M. Ashari and S. Anam, "A wide range fuzzy based maximum power point tracker for improving the efficiency and sizing of PV systems," Journal of Electrical Engineering, vol. 11, no. 2, p. 8, 2011.

[10] C. T. Kelley, Solving Nonlinear Equations with Newton's Method; Fundamentals of Algorithms, Society of Industrial and Applied Mathematics, 2003.

[11] http://www.ladyada.net/library/diyboostcalc.html.

[12] A. Garrigos and J. M. Blanes, "Power MOSFET is core of regulated-dc electronic load," University Miguel Hernandez, Orihuela, Spain, 2005, http://www.edn.com/. 

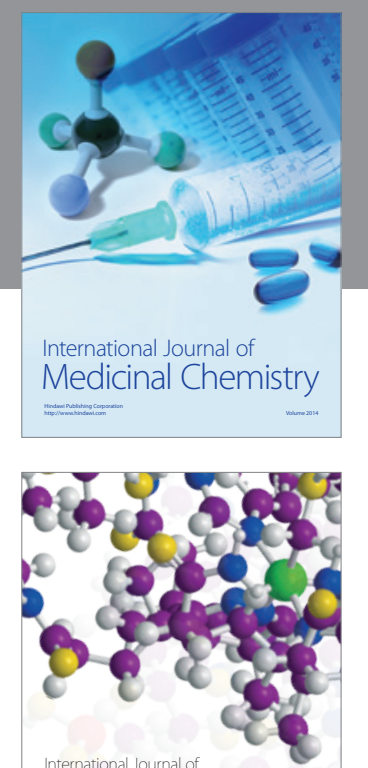

\section{Carbohydrate} Chemistry

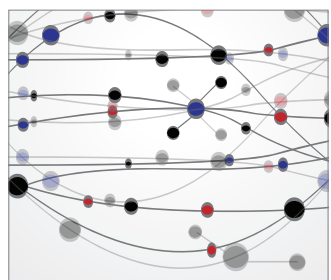

The Scientific World Journal
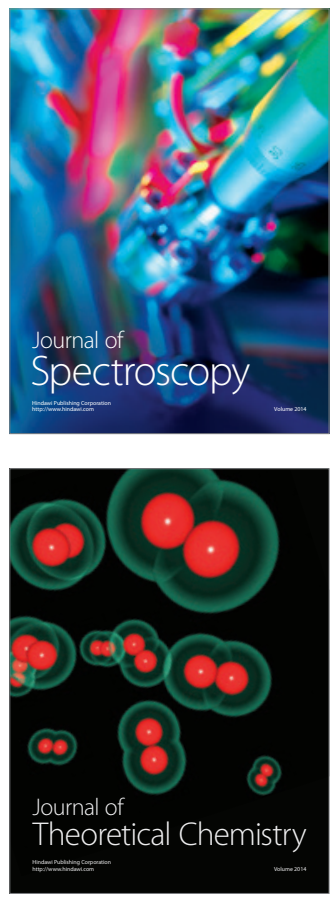
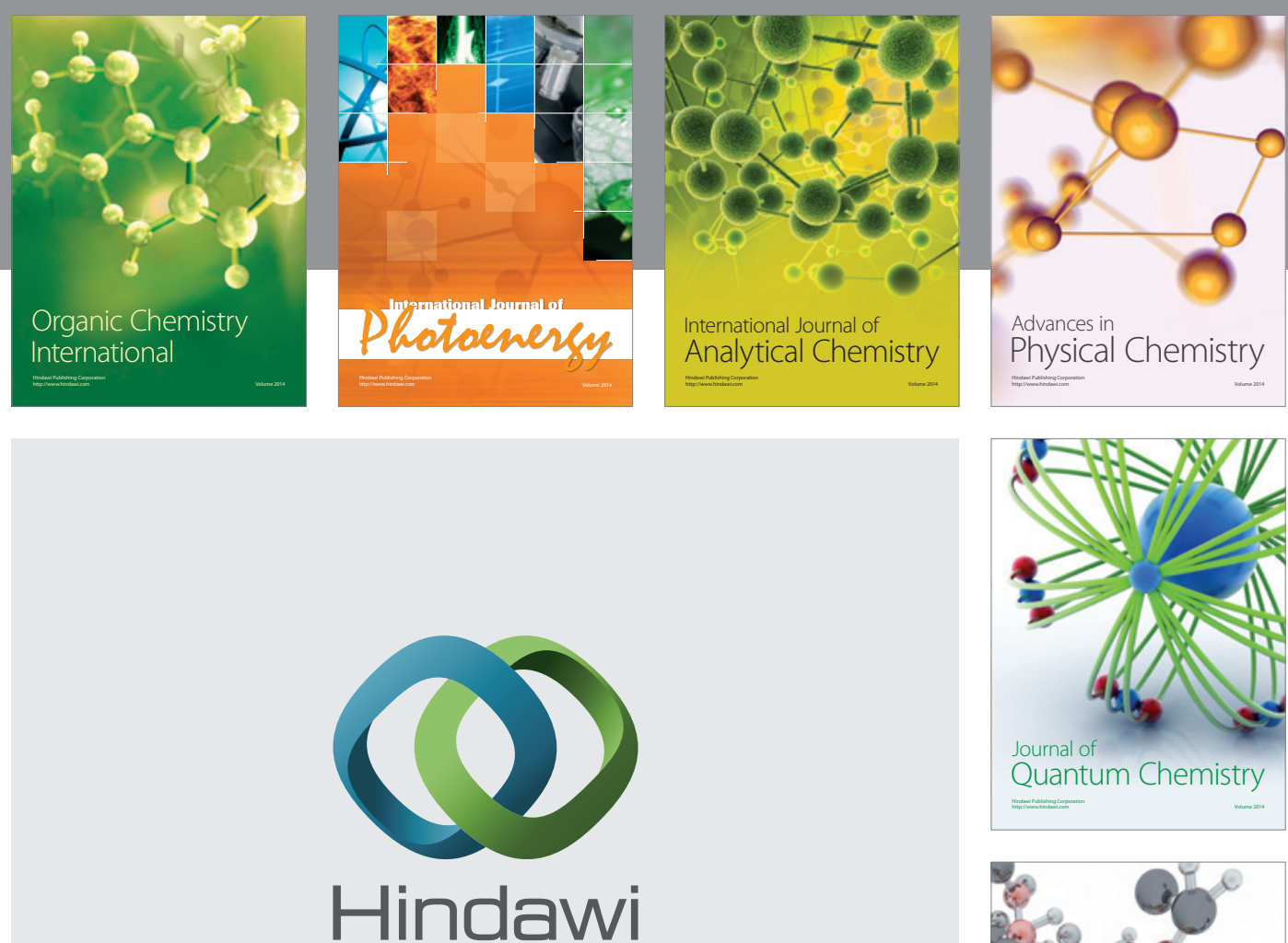

Submit your manuscripts at

http://www.hindawi.com

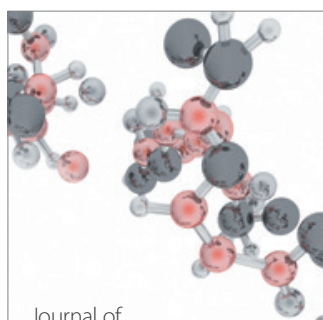

Analytical Methods

in Chemistry

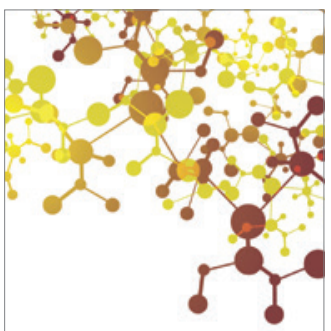

Journal of

Applied Chemistry

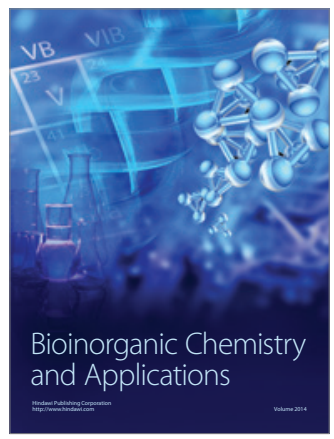

Inorganic Chemistry
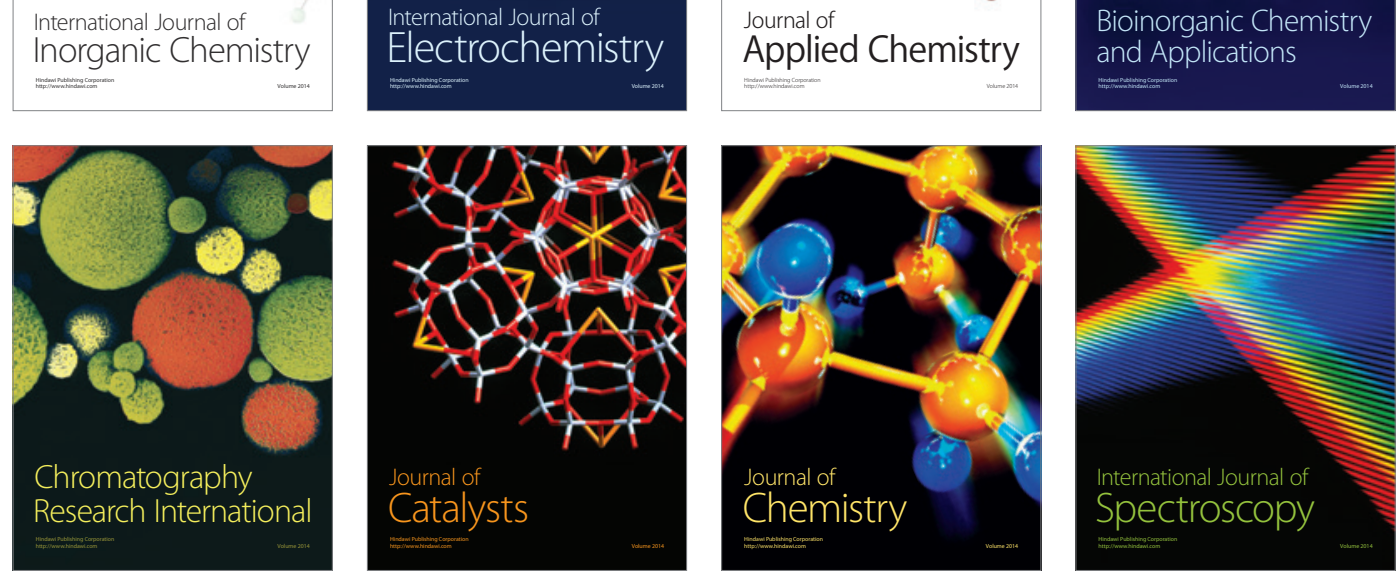